\title{
Reflets
}

Revue ontaroise d'intervention sociale et communautaire

\section{Lien : programme explorant le lien entre la violence et la toxicomanie}

\section{Julie Fournier}

Volume 2, numéro 1, printemps 1996

Contrer la violence subie par les femmes et les enfants en milieu familial

URI : https://id.erudit.org/iderudit/026116ar

DOI : https://doi.org/10.7202/026116ar

Aller au sommaire du numéro

Éditeur(s)

Reflets : Revue ontaroise d'intervention sociale et communautaire

ISSN

1203-4576 (imprimé)

1712-8498 (numérique)

Découvrir la revue

Citer cet article

Fournier, J. (1996). Lien : programme explorant le lien entre la violence et la toxicomanie. Reflets, 2(1), 174-177. https://doi.org/10.7202/026116ar

Tous droits réservés (C) Reflets : Revue ontaroise d'intervention sociale et communautaire, 1996
Ce document est protégé par la loi sur le droit d'auteur. L'utilisation des services d'Érudit (y compris la reproduction) est assujettie à sa politique d'utilisation que vous pouvez consulter en ligne.

https://apropos.erudit.org/fr/usagers/politique-dutilisation/ 


\section{Lien: programme explorant le lien entre la violence et la toxicomanie}

\section{Julie F ournier}

C onseillère en programmation, $\mathrm{F}$ ondation de la recherche sur la toxicomanie

La plupart du temps, l'existence d'un lien entre la violence et la toxicomanie ne surprend personne. Toutefois, on peut ignorer qu'il existe, en réalité, des liens et des mythes entourant ces deux phénomènes. Par exemple, les conseillers et conseillères des domaines de la toxicomanie et des services sociaux voient souvent des femmes qui vivent des relations de violence et qui soulagent leur douleur émotionnelle avec l'alcool ou d'autres stupéfiants. II y a leshommeset les femmesqui ont vécu de la violence physique, psychologique ou sexuelle dans leur enfance, et qui se réfugient dans l'alcool et les stupéfiants. M ais, il existe aussi un lien entre l'alcool et les personnes qui commettent les actes de violence. Bien que les buveurs excessifs aient plus tendance à être violents envers leur partenaire ou leurs enfants, les experts affirment que l'alcool n'engendre pas la violence, mais est un facteur qui la déclenche.

La trousse éducative $L$ ien aborde certaines questions reliées à la compréhension du problème. Lien a été mis au point par la Fondation de la recherche sur la toxicomanie (AR F), avec le soutien de Santé $C$ anada. La trousse permet de se documenter sur la toxicomanie et la violence faite aux femmes et aux enfants, en vue d'éliminer les mythes sur ces problèmes. $0 \mathrm{n}$ trouve des conseils pratiques destinés aux conseillers et conseillères en toxicomanie, au personnel des refuges pour femmes battues, aux intervenants et intervenantes des services de santé et sociaux. 
Les relations de violence et les toxicomanies créent des ensembles de problèmes qui coexistent et qui sont difficiles à dépister lorsque les clients et clientes s'adressent aux services d'intervention so ciale. A u C anada, il existe deux réseaux parallèles de traitement: I'un pour le traitement des toxicomanies, I'autre pour la violence familiale. II devient alors complexe, tant pour les fournisseurs de services que pour la clientèle, de faire le pont d'un traitement à l'autre. Par exemple, si une femme se trouve dans un refuge, le personnel peut ne pas voir les signes de toxicomanie, ou ne pas être préparé à traiter les problèmes découlant de l'usage d'alcool et de stupéfiants. D e même, un conseiller ou une conseillère en toxicomanie peut ne pas se rendre compte que l'usage de l'alcool chez une cliente prend racine dans la violence qu'elle a vécue dans son enfance ou qu'elle vit présentement.

«e programme $L$ ien permet aux intervenants et intervenantes de première ligne, ayant souvent affaire à des femmes et des enfants qui ont vécu la violence, de déceler les signes d'un usage possible d'alcool ou d'autres substancess, signale Julia G reenbaum, responsable de programme à la Fondation (ARF) et chef de l'équipe qui I'a élaboré. D e plus, « i ien sensibilise les conseillers et conseillères en toxicomanie aux symptômes de violence sexuelle et physique chez leurs clients et clientes. D ans chaque cas, il ou elle saura mieux déceler le problème et sera mieux en mesure de diriger la clientèle vers le service approprié», ajoute-t-elle.

En 1989, une étude menée par Groeneveld et Shain, de la Fondation (ARF), a révélé que les femmes qui ont vécu de la violence, soit dans leur enfance, soit à l'âge adulte, ont plus tendance à faire usage de stupéfiants pour calmer leur anxiété ou leur insomnie. Dans le cadre de l'étude, on a interrogé 2100 femmes vivant avec un homme. Les femmes qui subissent la violence sexuelle à l'âge adulte présentaient des pourcentages plus élevés d'usage de médicaments sur ordonnance ou en vente libre. Les femmes qui ont vécu la violence sexuelle dans leur enfance se plaçaient au deuxième rang. Ces dernières avaient deux fois plus tendance à faire usage de sédatifs, et environ $50 \%$ plus tendance que les femmes qui n'ont pas vécu la violence à faire usage 
de somnifères. L'usage de substancesillégales, comme la marijuana, le LSD, la cocaïne et les amphétamines, était beaucoup plus élevé chez les femmes ayant vécu une relation de violence.

Un rapport récent de Statistique C anada (1994) montre une pré valence semblable de la violence et de l'usage de drogues. Plus du quart desfemmes mariées qui ont vécu la violence trouvaient du réconfort dans l'alcool ou les stupéfiants. Finalement, les programmes de traitement de la toxicomanie à l'intention des femmes rapportent que jusqu'à $80 \%$ de leurs clientes ont vécu de la violence auparavant.

Publié en 1995, L ien a été mis au point par Judith G roeneveld pour parfaire les connaissances respectives des conseillers et conseillères en toxicomanie, ainsi que du personnel des refuges. Santé $C$ anada a commandité le projet afin d'en faire profiter tous les intervenants et intervenantes. En O ntario, les consultants et consultantes des programmes communautaires de la Fondation (AR F) font équipe avec les intervenants et intervenantes en violence familiale pour donner les ateliers destinés aux fournisseurs de services en toxicomanie, à l'aide à l'enfance, à la police, dans les programmes de travail social. C hacun desmodules de la trousse Lien est conçu de façon à donner aux fournisseurs de services, etc., des conseils précis sur les façons de poser les questions qui permettront de dépister \&'autre» problème. L'objectif est d'aider les fournisseurs de services à garder ce lien en tête lorsqu'ils rencontrent leursclients et clientes, et qu'ils s'informent sur cesquestions. $D$ 'autres modules contiennent des renseignements généraux sur la toxicomanie, la violence faite aux femmes et aux enfants, et les divers liens entre les deux questions. Finalement, L ien encourage aussi les fournisseurs de services à constituer des réseaux de partenaires dans leur propre collectivité, afin que la clientèle so it dirigée vers les services adéquats, et que chaque cas soit traité de manière appropriée.

\section{Note}

C et article a été écrit par $\mathrm{Craig}$ Smith, agent de communication de la Fondation de la recherche sur la toxicomanie àToronto en 
mai 1995 pour Le Journal. II a été révisé par Julie Fournier, Fondation de la recherche sur la toxicomanie, 201-383, avenue Parkdale, $\mathrm{O}$ ttawa, KIY 4R 4. Pour de plus amples renseignements sur $\mathrm{L}$ ien, veuillez communiquer avec Lise $\mathrm{N}$ olet au (416) $595-6632$

\section{Bibliographie}

GROENEVELD, J. et M. SH AIN (1989). T he domestic violence survey. A preliminary report, Toronto, Fondation de la recherche sur la toxicomanie.

GROENEVELD, J. et M. SHAIN (1989). D rug use among vidims of physical and sexual abuse: A preliminary report, Toronto, Fondation de la recherche sur la toxicomanie.

STATIST IQ UE CAN ADA (1994). R ésultats d'une enquête nationale sur l'agression contre la conjointe (catalogue no 85-002), 0 ttawa, M inistre des Approvisionnements et Services. 